\title{
Estimation of inequalities for warped product semi-slant submanifolds of Kenmotsu space forms
}

\author{
Misbah Liaqat ${ }^{1}$, Piscoran Laurian-Ioan², Wan Ainun Mior Othman ${ }^{3}$, Akram Ali $^{3^{*}} \mathbb{B}$, Abdullah Gani ${ }^{1}$ and \\ Cenap Ozel ${ }^{4,5}$
}

"Correspondence:

akramali133@gmail.com

${ }^{3}$ Institute of Mathematical Science,

Faculty of Sciences, University of

Malaya, Kuala Lumpur, 50603

Malaysia

Full list of author information is

available at the end of the article

\begin{abstract}
In this paper, we construct the geometric inequalities for the squared norm of the mean curvature and warping functions of warped product semi-slant submanifolds in Kenmotsu space forms. The equality cases are also discussed.
\end{abstract}

MSC: Primary 53C40; secondary 58C35; 53C55; 53C42; 53C15; 53D15

Keywords: warped products; semi-slant submanifold; inequalities; Kenmotsu space forms

\section{Introduction}

The theory of warped product manifolds is an emerging research area in differential geometry. The idea of a warped product manifold was first discovered by Bishop and O'Neil (cf. [1]) as a manifold of negative curvature. They defined the manifolds based on $M_{1}$ and $M_{2}$, which are the two Riemannian manifolds of dimensions $n_{1}$ and $n_{2}$ endowed with Riemannian matrices $g_{1}$ and $g_{2}$ such that $f: M_{1} \rightarrow(0, \infty)$ be a positive differentiable function on $M_{1}$. Thus, the warped product $M=M_{1} \times_{f} M_{2}$ is defined based on the product manifold $M_{1} \times M_{2}$ equipped with a metric $g=g_{1}+f^{2} \cdot g_{2}$. Moreover, if we consider that $\gamma_{1}: M_{1} \times M_{2} \rightarrow M_{1}$ and $\gamma_{2}: M_{1} \times M_{2} \rightarrow M_{2}$ are the natural projections on $M_{1}$ and $M_{2}$, respectively, then the metric $g$ on a warped product is defined as

$$
\|X\|^{2}=\left\|\gamma_{1}^{*}(X)\right\|^{2}+\left(f o \gamma_{1}\right)^{2}\left\|\gamma_{2}^{*}(X)\right\|^{2},
$$

for any $X$ tangent to TM. The function $f$ is called the warping function. If $f=1$, then $M$ is called a simply Riemannian product manifold. In contrast, $M$ is denoted a non-trivial warped product manifold when $f \neq 1$. Let $M=M_{1} \times_{f} M_{2}$ be a non-trivial warped product manifold of an arbitrary Riemannian manifold $\widetilde{M}$. Then

$$
\nabla_{X} Z=\nabla_{Z} X=(X \ln f) Z,
$$

for any vector fields $X \in \Gamma\left(T M_{1}\right)$ and $Z \in \Gamma\left(T M_{2}\right)$. Further, $\nabla$ is a Levi-Citvita connection of the induced Riemannian manifold $M$.

(c) 2016 Liaqat et al. This article is distributed under the terms of the Creative Commons Attribution 4.0 International License (http://creativecommons.org/licenses/by/4.0/), which permits unrestricted use, distribution, and reproduction in any medium, provided you give appropriate credit to the original author(s) and the source, provide a link to the Creative Commons license, and indicate if changes were made. 
The approach of such type inequalities for warped products in almost Hermitian and almost contact metric manifolds has been an important field for a few decades. Especially, Chen in [2] obtained the sharp relationship between norm of the squared mean curvature and the warping function $f$ of the warped product $M_{1} \times_{f} M_{2}$ isometrically immersed in a real space form, i.e., we have the following.

Theorem 1.1 Let $\phi: M_{1} \times_{f} M_{2}$ be an isometrically immersion of an n-dimensional warped product into $2 m$-dimensional real space form $\widetilde{M}(c)$ with constant sectional curvature $c$. Then

$$
\frac{\Delta f}{f} \leq \frac{n^{2}}{4 n_{2}}\|H\|^{2}+n_{1} \cdot c
$$

where $n_{i}=\operatorname{dim} M_{i}, i=1,2$, and $\nabla$ is the Lapalcian operator of $M_{1}$. Moreover, the equality holds in the above if and only if $\phi$ is a mixed totally geodesic and $n_{1} H_{1}=n_{2} H_{2}$ such that $H_{1}$ and $\mathrm{H}_{2}$ are partial mean curvatures.

However, on the base of the literature, we find that several inequalities have been extended to various structures for warped products by many geometers in [3-9]. Therefore other inequalities also appear, in [4, 5, 10-19] for slant submanifolds and semi-slant submanifolds in different curvature forms, which are called Chen inequalities. In addition, it is well known that Atceken [20] studied the non-existence of the warped product semislant submanifolds of a Kenmotsu manifold such that structure vector $\xi$ is tangent to the fiber. Meanwhile, Uddin in [21] and Srivastava in [22] proved that the warped product semi-slant submanifold of a Kenmotsu manifold exists in the forms $M=M_{T} \times_{f} M_{\theta}$ and $M=M_{\theta} \times_{f} M_{T}$, except in the case when the structure vector field $\xi$ is tangent to $M_{T}$ and $M_{\theta}$, respectively. Moreover, we have studied some inequalities that Cioroboiu [13] and Aktan et al. [12] obtained for semi-slant submanifolds by constructing its orthonormal frame but overlooking the suitable conditions for inequalities of a warped semi-slant product. Therefore, one needs to derive the inequalities for the mean curvature and warping functions with slant angles of a warped semi-slant product in Kemotsu space form. In the current paper, we are extending studies like [13] for warped product semi-slant submanifolds in a Kenmotsu space form. We also generalize some other inequalities for CR-warped product submanifolds in special cases because of the warped product of semi-slant generalized CR-warped products in Kenmotsu manifolds. Moreover, the equality cases and geometric inequalities applications related to Wireless Sensor Network are also discussed.

\section{Preliminaries}

An odd $(2 m+1)$-dimensional smooth manifold $\widetilde{M}$ is called a Kenmotsu manifold, if it is consisting in an endomorphism $\varphi$ of its tangent bundle $T \widetilde{M}$, a structure vector field $\xi$, and a 1-form $\eta$ satisfying the following:

$$
\begin{aligned}
& \varphi^{2}=-I+\eta \oplus \xi, \quad \eta(\xi)=1, \quad \eta \circ \varphi=0, \\
& g(\varphi U, \varphi V)=g(U, V)-\eta(U) \eta(V), \quad \eta(U)=g(U, \xi),
\end{aligned}
$$

and the structure equation is given by

$$
\left(\widetilde{\nabla}_{U} \varphi\right) V=g(\varphi U, V)-\eta(V) \varphi U \text {, }
$$




$$
\widetilde{\nabla}_{U} \xi=U-\eta(U) \xi
$$

for any $U, V$ tangent to $\widetilde{M}$ (see [23]). The curvature tensor $\widetilde{R}$ for Kenmotsu space forms is defined as

$$
\begin{aligned}
\widetilde{R}(X, Y, Z, W)= & \frac{c-3}{4}\{g(X, W) g(Y, Z)-g(X, Z) g(Y, W)\} \\
& +\frac{c+1}{4}\{g(X, \varphi W) g(Y, \varphi Z)-g(X, \varphi Z) g(Y, \varphi W) \\
& -2 g(X, \varphi Y) g(Z, \varphi W)-g(X, W) \eta(Y) \eta(Z) \\
& +g(X, Z) \eta(X) \eta(W)-g(Y, Z) \eta(X) \eta(W) \\
& +g(Y, W) \eta(X) \eta(Z)\},
\end{aligned}
$$

where $c$ is a function of the constant $\varphi$-sectional curvature of $\widetilde{M}$ (see [3].

Let $M$ be a submanifold of an almost contact metric manifold $\widetilde{M}$ with induced metric $g$; if $\nabla$ and $\nabla^{\perp}$ are the induced connections on the tangent bundle $T M$ and the normal bundle $T^{\perp} M$ of $M$, respectively, then the Gauss and Weingarten formulas are given by

$$
\begin{aligned}
& \widetilde{\nabla}_{U} V=\nabla_{U} V+h(U, V), \\
& \widetilde{\nabla}_{U} N=-A_{N} U+\nabla_{U}^{\perp} N,
\end{aligned}
$$

for each $U, V \in \Gamma(T M)$ and $N \in \Gamma\left(T^{\perp} M\right)$, where $h$ and $A_{N}$ are the second fundamental form and the shape operator (corresponding to the normal vector field $N$ ), respectively, for the immersion of $M$ into $\widetilde{M}$. They are related as

$$
g(h(U, V), N)=g\left(A_{N} U, V\right),
$$

where $g$ denotes the Riemannian metric on $\widetilde{M}$ as well as the metric induced on $M$. Moreover, for a submanifold $M$, the Gauss equation is defined as

$$
\begin{aligned}
\widetilde{R}(U, V, Z, W)= & R(U, V, Z, W)+g(h(U, Z), h(V, W)) \\
& -g(h(U, W), h(V, Z)),
\end{aligned}
$$

for any $U, V, Z, W \in \Gamma(T M)$, where $\widetilde{R}$ and $R$ are the curvature tensors on $\widetilde{M}$ and $M$, respectively. The mean curvature vector $H$ for an orthonormal frame $\left\{e_{1}, e_{2}, \ldots, e_{n}\right\}$ of the tangent space $T M$ on $M$ is defined by

$$
H=\frac{1}{n} \operatorname{trace}(h)=\frac{1}{n} \sum_{i=1}^{n} h\left(e_{i}, e_{i}\right),
$$

where $n=\operatorname{dim} M$. In addition, we set

$$
h_{i j}^{r}=g\left(h\left(e_{i}, e_{j}\right), e_{r}\right) \quad \text { and } \quad\|P\|^{2}=\sum_{i, j=1}^{n} g^{2}\left(\varphi e_{i}, e_{j}\right)
$$


Furthermore, the scalar curvature $\rho$ for a submanifold $M$ of an almost contact manifold $\widetilde{M}$ is given by

$$
\rho=\sum_{1 \leq i \neq j \leq n} K\left(e_{i} \wedge e_{j}\right)
$$

where $K\left(e_{i} \wedge e_{j}\right)$ is the sectional curvature of plane section spanned by $e_{i}$ and $e_{j}$. Let $G_{r}$ be a $r$-plane section on $T M$ and $\left\{e_{1}, e_{2}, \ldots, e_{r}\right\}$ any orthonormal basis of $G_{r}$. Then the scalar curvature $\rho\left(G_{r}\right)$ of $G_{r}$ is given by

$$
\rho\left(G_{r}\right)=\sum_{1 \leq i \neq j \leq r} K\left(e_{i} \wedge e_{j}\right)
$$

Let $\widetilde{M}$ be a Kenmotsu manifold with an almost contact structure $(\varphi, \xi, \eta)$ and $M$ be a submanifold tangent to the structure vector field $\xi$ isometrically immersed in $\widetilde{M}$. Then $M$ is called invariant if $\varphi\left(T_{p} M\right) \subseteq T_{p} M$, and $M$ is called anti-invariant if $\varphi\left(T_{p} M\right) \subset T_{p}^{\perp} M$ for every $p \in M$ where $T_{p} M$ denotes the tangent bundle of $M$ at the point $p$. Moreover, $M$ is called a slant submanifold if all non-zero vectors $U$ tangent to $M$ at a point $p$, the angle of $\theta(U)$ between $\varphi U$ and $T_{p} M$ are constant, i.e., they do not depend on the choice of $p \in M$ and $U \in \Gamma\left(T_{p} M-\langle\xi(p)\rangle\right)$ (see [24]). Except invariant, anti-invariant, and slant submanifolds, there are several other classes of submanifolds determined by the behavior of the tangent space of the submanifold under the action of a one-one tensor field $\varphi$ of an ambient manifold.

Recently, Cabrerizo et al. [24] extended of the mentioned definition into a characterization for a slant submanifold in a contact metric manifold. In fact, they have obtained the following theorem.

Theorem 2.1 Let $M$ be a submanifold of an almost contact metric manifold $\widetilde{M}$ such that $\xi \in T M$. Then $M$ is slant if and only if there exists a constant $\lambda \in[0,1]$ such that

$$
P^{2}=\delta(-I+\eta \otimes \xi)
$$

Furthermore, in such a case, if $\theta$ is a slant angle, then it satisfies $\delta=\cos ^{2} \theta$.

Hence, we have the following relations which are consequences of Theorem 2.1, i.e.,

$$
\begin{aligned}
& g(P U, P V)=\cos ^{2} \theta\{g(U, V)-\eta(U) \eta(V)\}, \\
& g(F U, F V)=\sin ^{2} \theta\{g(U, V)-\eta(U) \eta(V)\} .
\end{aligned}
$$

There is another class, which is called a semi-slant submanifold. The notion of semislant submanifolds were defined and studied by Papaghiuc in [25] as a natural generalization of CR-submanifolds of almost Hermitian manifolds in terms of the slant distribution and it was later extended to the setting of contact manifolds by Cabrerizo [26]. One defined these submanifolds as follows.

Definition 2.2 Let $M$ be a submanifold of an almost contact metric manifold $\widetilde{M}$. Then $M$ is said to be a semi-slant submanifold if there exist two orthogonal distributions $\mathcal{D}$ and $\mathcal{D}^{\theta}$ such that 
(i) $T M=\mathcal{D} \oplus \mathcal{D}^{\theta} \oplus\langle\xi\rangle$ where $\langle\xi\rangle$ is a 1-dimensional distribution spanned by $\xi$ :

(ii) $\mathcal{D}$ is invariant, i.e., $\varphi(\mathcal{D}) \subseteq \mathcal{D}$;

(iii) $\mathcal{D}^{\theta}$ is slant distribution with slant angle $\theta \neq 0, \pi / 2$.

Assume that $\phi: M=M_{1} \times_{f} M_{2} \rightarrow \tilde{M}$ is an isometric immersion of a warped product $M_{1} \times_{f} M_{2}$ into a Riemannian manifold of $\tilde{M}$ of constant section curvature $c$. Suppose that $n_{1}, n_{2}$, and $n$ are the dimensions of $M_{1}, M_{2}$, and $M_{1} \times_{f} M_{2}$, respectively. Then for unit vector fields $X, Z$ tangent to $M_{1}, M_{2}$, respectively,

$$
\begin{aligned}
K(X \wedge Z) & =g\left(\nabla_{Z} \nabla_{X} X-\nabla_{X} \nabla_{Z} X, Z\right) \\
& =\frac{1}{f}\left\{\left(\nabla_{X} X\right) f-X^{2} f\right\} .
\end{aligned}
$$

Let us assume a local orthonormal frame $\left\{e_{1}, e_{2}, \ldots, e_{n}\right\}$ such that $e_{1}, e_{2}, \ldots, e_{n_{1}}$ tangent to $M_{1}$ and $e_{n_{1}+1}, \ldots, e_{n}$ are tangent to $M_{2}$. Then

$$
\sum_{1 \leq i \leq n_{1}} \sum_{n_{1}+1 \leq j \leq n} K\left(e_{i} \wedge e_{j}\right)=\frac{n_{2} \cdot \Delta f}{f}
$$

Lemma 2.3 [27] Let $a_{1}, a_{2}, \ldots, a_{n}, a_{n+1}$ be $n+1(n \geq 2)$ be real number such that

$$
\left(\sum_{i=1}^{n} a_{i}\right)^{2}=(n-1)\left(\sum_{i=1}^{n} a_{i}^{2}+a_{n+1}\right)
$$

Then $2 a_{1} \cdot a_{2} \geq a_{3}$ with the equality holding if and only if $a_{1}+a_{2}=a_{3}=\cdots=a_{k}$.

\section{Main inequalities}

In this section, as applications of very famous studied of Nolker in [28], we obtain the following inequality for warped product semi-slant submanifolds of Kenmotsu space form such that $\xi$ is tangent to the first factor of the warped product, i.e., we have the following.

Theorem 3.1 Assume that $\phi: M=M_{T} \times_{f} M_{\theta} \rightarrow \tilde{M}(c)$ is an isometric immersion from a warped product semi-slant $M_{T} \times_{f} M_{\theta}$ into a Kenmotsu space form $\tilde{M}(c)$ such that $c$ is a $\varphi$-sectional constant curvature and $\xi$ is tangent to $M_{T}$. Then:

(i) The relation between warping function and the squared norm of mean curvature is obtained

$$
\frac{\Delta f}{f} \leq \frac{n^{2}}{4 n_{2}}\|H\|^{2}+\frac{c-3}{4} n_{1}-\frac{c+1}{4 n_{2}}\left[3 d_{1}+d_{2}\left(2+3 \cos ^{2} \theta\right)\right]
$$

where $n_{i}=\operatorname{dim} M_{i}, i=T, \theta$, and $\Delta$ is the Laplacian operator on $M_{T}$.

(ii) The equality case holds in (3.1) if and only if $n_{1} \cdot H_{T}=n_{2} \cdot H_{\theta}$, where $H_{T}$ and $H_{\theta}$ are partially mean curvature vectors on $M_{T}$ and $M_{\theta}$, respectively. Moreover, $\phi$ is a mixed totally geodesic immersion. 
Proof Let $M_{T} \times_{f} M_{\theta}$ be a warped product semi-slant submanifold in Kenmotsu space form $\tilde{M}(c)$. Based on the Gauss formula (2.9) and (2.5), we derive

$$
\begin{aligned}
2 \rho= & \frac{c-3}{4} n(n-1)+\frac{(c+1)}{4}\left[3 \sum_{1 \leq i \neq k \leq n} g^{2}\left(P e_{i}, e_{k}\right)-2(n-1)\right] \\
& +n^{2}\|H\|^{2}-\|h\|^{2} .
\end{aligned}
$$

As well we are concerned that $M$ is a proper semi-slant submanifold of Kenmotsu space form $\widetilde{M}(c)$. Thus, we define the following frame according to Cioroboiu in [13], i.e.,

$$
\begin{aligned}
e_{1}, e_{2} & =\varphi e_{1}, \ldots, e_{2 d_{1}-1}, e_{2 d_{1}}=\varphi e_{2 d_{1}-1}, \\
e_{2 d_{1}+1}, e_{2 d_{1}+2} & =\sec \theta P e_{2 d_{1}+1}, \ldots \quad e_{2 d_{1}+2 d_{2}-1}, e_{2 d_{1}+2 d_{2}}=\sec \theta P e_{2 d_{1}+2 d_{2}-1}, \\
e_{2 d_{1}+2 d_{2}+1} & =\xi
\end{aligned}
$$

Clearly, we derive

$$
g^{2}\left(\varphi e_{i}, e_{i+1}\right)= \begin{cases}1, & \text { for } i \in\left\{1,2, \ldots, 2 d_{1}-1\right\} \\ \cos ^{2} \theta, & \text { for } i \in\left\{2 d_{1}+1, \ldots, 2 d_{1}+2 d_{2}-1\right\}\end{cases}
$$

From (3.2) and (3.3), it follows that

$$
\begin{aligned}
2 \rho= & \frac{c-3}{4} n(n-1)+\frac{(c+1)}{4}\left[d_{1}+d_{2} \cdot\left(3 \cos ^{2} \theta-2\right)\right] \\
& +n^{2}\|H\|^{2}-\|h\|^{2} .
\end{aligned}
$$

Now we consider

$$
\delta=2 \rho-\frac{c-3}{4} n(n-1)-\frac{(c+1)}{2}\left[d_{1}+d_{2} \cdot\left(3 \cos ^{2} \theta-2\right)\right]-\frac{n}{2}\|H\|^{2} .
$$

Then, from (3.4) and (3.5),

$$
n^{2}\|H\|^{2}=2\left(\delta-\|h\|^{2}\right)
$$

Thus for a locally orthonormal frame $\left\{e_{1}, e_{2}, \ldots, e_{n}\right\}$, equation (3.6) takes the form

$$
\left(\sum_{r=n+1}^{2 m+1} \sum_{i=1}^{n} h_{i i}^{r}\right)^{2}=2\left(\delta+\sum_{r=n+1}^{2 m+1} \sum_{i=1}^{n}\left(h_{i i}^{r}\right)^{2}+\sum_{r=n+1}^{2 m+1} \sum_{i<j=1}^{n}\left(h_{i j}^{r}\right)^{2}+\sum_{r=n+1}^{2 m+1} \sum_{i, j=1}^{n}\left(h_{i j}^{r}\right)^{2}\right),
$$

which implies that

$$
\begin{aligned}
\frac{1}{2}\left(h_{11}^{n+1}+\sum_{i=2}^{n_{1}} h_{i i}^{n+1}+\sum_{t=n_{1}+1}^{n} h_{t t}^{n+1}\right)^{2}= & \delta\left(h_{11}^{n+1}\right)^{2}+\sum_{i=2}^{n_{1}}\left(h_{i i}^{n+1}\right)^{2} \\
& +\sum_{t=n_{1}+1}^{n}\left(h_{t t}^{n+1}\right)^{2}-\sum_{2 \leq j \neq l \leq n_{1}} h_{j j}^{n+1} h_{l l}^{n+1}
\end{aligned}
$$




$$
\begin{aligned}
& -\sum_{n_{1}+1 \leq t \neq s \leq n} h_{t t}^{n+1} h_{s s}^{n+1}+\sum_{i<j=1}^{n}\left(h_{i j}^{n+1}\right)^{2} \\
& +\sum_{r=n+1}^{2 m+1} \sum_{i, j=1}^{n}\left(h_{i j}^{r}\right)^{2} .
\end{aligned}
$$

Now consider that $a_{1}=h_{11}^{n+1}, a_{2}=\sum_{i=2}^{n_{1}} h_{i i}^{n+1}$, and $a_{3}=\sum_{t=n_{1}+1}^{n} h_{t t}^{n+1}$ and applying Lemma 2.3 in (3.8). Then we derive

$$
\frac{\delta}{2}+\sum_{i<j=1}^{n}\left(h_{i j}^{n+1}\right)^{2}+\frac{1}{2} \sum_{r=n+1}^{2 m+1} \sum_{i, j=1}^{n}\left(h_{i j}^{r}\right)^{2} \leq \sum_{2 \leq j \neq l \leq n_{1}} h_{j j}^{n+1} h_{l l}^{n+1}+\sum_{n_{1}+1 \leq t \neq s \leq n} h_{t t}^{n+1} h_{s s}^{n+1},
$$

with equality holding in (3.9) if and only if

$$
\sum_{i=1}^{n_{1}} h_{i i}^{n+1}=\sum_{t=n_{1}+1}^{n} h_{t t}^{n+1}
$$

Further, (2.12) and (2.18) imply that

$$
\frac{n_{2} \cdot \Delta f}{f}=\rho-\sum_{1 \leq j \neq k \leq n_{1}} K\left(e_{i} \wedge e_{k}\right)-\sum_{n_{1}+1 \leq t \neq s \leq n} K\left(e_{t} \wedge e_{s}\right)
$$

From (2.5) we obtain

$$
\begin{aligned}
\frac{n_{2} \cdot \Delta f}{f}= & \rho-\frac{c-3}{8} n_{1}\left(n_{1}-1\right)+\frac{(c+1)}{4}\left(n_{1}-1\right) \\
& -\frac{3(c+1)}{4} \sum_{1 \leq i \neq k \leq n_{1}} g^{2}\left(P e_{i}, e_{k}\right)-\sum_{r=1}^{2 m+1} \sum_{2 \leq j \neq k \leq n_{1}}\left(h_{j j}^{r} h_{k k}^{r}-\left(h_{j k}^{r}\right)^{2}\right) \\
& -\frac{3(c+1)}{4} \sum_{n_{1}+1 \leq t \neq s \leq n} g^{2}\left(P e_{t}, e_{s}\right)-\frac{c-3}{8} n_{2}\left(n_{2}-1\right) \\
& -\sum_{r=1}^{2 m+1} \sum_{n_{1}+1 \leq t \neq s \leq n}\left(h_{t t}^{r} h_{s s}^{r}-\left(h_{t s}^{r}\right)^{2}\right) .
\end{aligned}
$$

Thus, from (3.9) and (3.12), it is easily observed that

$$
\begin{aligned}
\frac{n_{2} \cdot \Delta f}{f} \leq & \rho-\frac{c-3}{8} n(n-1)+\frac{c-3}{4} n_{1} \cdot n_{2}+\frac{c+1}{4}\left(n_{1}-1\right) \\
& -\frac{3(c+1)}{4}\left(n_{1}-1\right)-\frac{3(c+1)}{4} n_{2} \cdot \cos ^{2} \theta-\frac{\delta}{2} .
\end{aligned}
$$

Hence, using (3.6), then the inequality (3.13) reduces to

$$
\frac{n_{2} \cdot \Delta f}{f} \leq \frac{n^{2}}{4}\|H\|^{2}+\frac{c-3}{4} n_{1} \cdot n_{2}+\frac{c+1}{4}\left(-3 d_{1}-3 d_{2} \cos ^{2} \theta-2 d_{2}\right) .
$$


This implies the inequality (3.1). The equality sign holds in (3.1) if and only if the terms left in (3.9), and (3.10) imply that

$$
\sum_{r=n+2}^{2 m+1} \sum_{j=1}^{n_{1}} h_{j j}^{r}=\sum_{r=n+2}^{2 m+1} \sum_{i=n_{1}+1}^{n} h_{i i}^{r}=0,
$$

and $n_{1} \cdot H_{T}=n_{2} \cdot H_{\theta}$, where $H_{T}$ and $H_{\theta}$ are partially mean curvature vectors on $M_{T}$ and $M_{\theta}$, respectively. Moreover, from (3.9), we find that

$$
h_{i j}^{r}=0, \quad \text { for each } 1 \leq i \leq n_{1}, n_{1}+1 \leq j \leq n, n+1 \leq r \leq 2 m+1 .
$$

It means that $\phi$ is a mixed totally geodesic immersion. But the converse of (3.16) may not be true in a warped semi-slant product in Kenmotsu space form. Thus the proof of the theorem is completed.

Theorem 3.2 Let $\phi: M=M_{\theta} \times_{f} M_{T} \rightarrow \widetilde{M}(c)$ be an isometric immersion of a warped product semi-slant $M_{\theta} \times_{f} M_{T}$ into a Kenmotsu space form $\widetilde{M}(c)$ such that $\xi$ is tangent to $M_{\theta}$. Then

(i) The relation between warping function and the norm of the squared mean curvature is given by

$$
\frac{\Delta f}{f} \leq \frac{n^{2}}{4 n_{2}}\|H\|^{2}+\frac{c-3}{4} n_{1}-\frac{c+1}{4 n_{2}}\left(3 d_{2}+d_{1}\left\{2+3 \cos ^{2} \theta\right\}\right)
$$

where $n_{i}=\operatorname{dim} M_{i}, i=T, \theta$, and $\Delta$ is the Laplacian operator on $M_{\theta}$.

(ii) The equality case holds in (3.17) if and only if $n_{1} \cdot H_{T}=n_{2} \cdot H_{\theta}$, where $H_{T}$ and $H_{\theta}$ are partially mean curvature vector fields on $M_{T}$ and $M_{\theta}$, respectively, and $\phi$ is a mixed totally geodesic immersion.

Proof The proof of Theorem 3.2 is similar to Theorem 3.1 by reversing and considering that the structure vector field $\xi$ is normal to the fiber.

In the sense of Papaghiuc, i.e., the generalization of semi-slant submanifolds, we directly obtain the following corollaries by using Theorem 3.1, Theorem 3.2, and $\theta=\frac{\pi}{2}$.

Corollary 3.3 Assume that $\phi: M=M_{T} \times_{f} M_{\perp} \rightarrow \widetilde{M}(c)$ is an isometric immersion of a CRwarped product $M_{T} \times_{f} M_{\perp}$ into a Kenmotsu space form $\widetilde{M}(c)$ with $c$ a $\varphi$-sectional constant curvature such that $\xi$ is tangent to $M_{T}$. Then

$$
\frac{\Delta f}{f} \leq \frac{n^{2}}{4 n_{2}}\|H\|^{2}+\frac{c-3}{4} n_{1}-\frac{c+1}{4 n_{2}}\left(3 d_{1}+2 d_{2}\right)
$$

where $n_{i}=\operatorname{dim} M_{i}, i=T, \perp$, and $\Delta$ is the Laplacian operator on $M_{T}$.

Corollary 3.4 Let $\phi: M=M_{\perp} \times_{f} M_{T} \rightarrow \widetilde{M}(c)$ be an isometric immersion of a CR-warped product submanifold $M_{\perp} \times_{f} M_{T}$ into a Kenmotsu space form $\tilde{M}(c)$ such that $\xi$ is tangent 
to $M_{\perp}$. Then

$$
\frac{\Delta f}{f} \leq \frac{n^{2}}{4 n_{2}}\|H\|^{2}+\frac{c-3}{4} n_{1}-\frac{c+1}{4 n_{2}}\left(3 d_{2}+2 d_{1}\right)
$$

where $n_{i}=\operatorname{dim} M_{i}, i=T, \perp$, and $\Delta$ is the Laplacian operator on $M_{\perp}$.

\section{Applications}

Any geometric inequality reflects a free or constrained optimum problem with suitable strategies for improved bandwidth management in wireless communications due to the dynamically changing traffic conditions and network performance. Except, some applications of geometric inequalities can be found in Wireless Sensor Networks related to Power balanced coverage-time optimization and Coverage by randomly deployed sensors [29]. Therefore, some applications of geometric inequalities can be found in computer sciences.

Competing interests

The authors declared that they have no competing interests.

Authors' contributions

All authors have equally contributed in this work. All authors read and approved the final manuscript.

\section{Author details}

${ }^{1}$ Center for Mobile Cloud Computing Research Lab (C4MCCR), Faculty of Computer Science and Information Technology, University of Malaya, Kuala Lumpur, 50603, Malaysia. ${ }^{2}$ Department of Mathematics and Computer Science, North University Center of Baia Mare, Technical University of Cluj Napoca, Victoriei 76, Baia Mare, 430122, Romania. ${ }^{3}$ Institute of Mathematical Science, Faculty of Sciences, University of Malaya, Kuala Lumpur, 50603, Malaysia. ${ }^{4}$ Department of Mathematics, Faculty of Sciences, King Abdul Aziz University, Jeddah, Saudi Arabia. ${ }^{5}$ Department of Mathematics, Faculty of Science, Dokuz Eylul University, Izmir, Turkey.

\section{Acknowledgements}

This work is funded by of the Malaysian Ministry of Education under the High Impact Research Grant no. UM.C/HIR/625/1/MOE/FSIT/03. The corresponding author supported by UMRG grant no. FP074-2015A.

Received: 22 May 2016 Accepted: 16 September 2016 Published online: 29 September 2016

\section{References}

1. Bishop, RL, O'Neil, B: Manifolds of negative curvature. Trans. Am. Math. Soc. 145, 1-9 (1969)

2. Chen, BY: On isometric minimal immersion from warped product submanifolds into real space forms. Proc. Edinb. Math. Soc. 45, 579-587 (2002)

3. Murathan, C, Arslan, K, Ezentas, R, Mihai, I: Warped product submanifolds in Kenmotsu space forms. Taiwan. J. Math. 1, 1431-1441 (2006)

4. Mihai, A: B. Y. Chen inequalities for slant submanifolds in generalized complex space forms. Rad. Mat. 12, 215-231 (2004)

5. Shukla, SS, Rao, PK: B. Y. Chen inequalities for bi-slant submanifolds in generalized complex space forms. J. Nonlinear Sci. Appl. 3, 283-292 (2010)

6. Uddin, S, Alqahtani, LS: Chen type inequality for warped product immersions in cosymplectic space forms. J. Nonlinear Sci. Appl. 9, 2914-2921 (2016)

7. Yoon, DW: Some inequalities of warped product submanifolds of cosymplectic space forms. Differ. Geom. Dyn. Syst. 6, 55-58 (2004)

8. Yoon, DW, Cho, KS, Han, SG: Some inequalities of warped products of locally conformal almost cosymplectic manifolds. Note Mat. 23(1), 51-60 (2004)

9. Atceken, M: Contact CR-warped product submanifolds in Sasakian space forms. Hacet. J. Math. Stat. 44(1), 23-32 (2015)

10. Ali, A, Othman, WAM, Ozel, C: Some inequalities for warped product pseudo-slant submanifolds of nearly Kenmotsu manifolds. J. Inequal. Appl. 2015, Article ID 9 (2015)

11. Ali, A, Uddin, S, Othman, WAM, Laurian-Ioan, P, Ozel, C: Curvature inequalities for C-totally real doubly warped product of locally conformal almost cosymplectic manifolds. Filomat (2016, in press)

12. Aktan, N, Sarikaya, MZ, Ozusaglam, E: B. Y. Chen's inequality for semi-slant submanifolds in T-space forms. Balk. J. Geom. Appl. 13(1), 1-10 (2008)

13. Cioroboiu, D: B. Y. Chen inequalities for semi-slant submanifolds of Sasakian space forms. Int. J. Math. Math. Sci. 27, 1731-1738 (2003)

14. Cioroboiu, D, Oiaga, A: B. Y. Chen inequalities for slant submanifolds in Sasakian space forms. Rend. Circ. Mat. Palermo $10,367-381(2004)$ 
15. Lee, CW, Lee, JW, Won, J, Vilcu, G-E: A new proof for some optimal inequalities involving generalized normalized $\delta$-Casorati curvatures. J. Inequal. Appl. 2015, Article ID 310 (2015)

16. Lee, CW, Yoon, DW, Lee, JW: Optimal inequalities for the Casorati curvatures of submanifolds of real space forms endowed with semi-symmetric metric connections. J. Inequal. Appl. 2014, Article ID 327 (2014)

17. Ozgur, C, Murathan, C: Chen inequalities for submanifolds of a locally conformal almost cosymplectic manifolds with semi-symmetric metric connection. An. Stiint. Univ. 'Ovidius' Constanța 18(1), 239-254 (2001)

18. Vilcu, G-E: On Chen invariants and inequalities in quaternionic geometry. J. Inequal. Appl. 2013, Article ID 66 (2013)

19. Dirik, S, Atçeken, M, Yildirim, Ü: Pseudo-slant submanifold in Kenmotsu space forms. J. Adv. Math. 11, 5680-5696 (2016)

20. Atceken, M: Warped product semi-slant submanifolds in Kenmotsu manifolds. Turk. J. Math. 34, 425-432 (2010)

21. Uddin, S, Khan, VA, Khan, KA: Warped product submanifolds of Kenmotsu manifolds. Turk. J. Math. 36, 319-330 (2012)

22. Srivastava, SK: Warped product submanifolds of Kenmotsu manifolds. arXiv:1206.4416V2 [math.DG] 26 Jan 2013

23. Kenmotsu, K: A class of almost contact Riemannian manifolds. Tohoku Math. J. 24, 93-103 (1972)

24. Cabrerizo, JL, Carriazo, A, Fernandez, LM, Fernandez, M: Slant submanifolds in Sasakian manifolds. Glasg. Math. J. 42, 125-138 (2000)

25. Papaghiuc, N: Semi-slant submanifold of Kaehlerian manifold. An. Ştiinţ. Univ. 'Al.I. Cuza' Iaşi, Mat. 40, 55-61 (1994)

26. Cabrerizio, JL, Carriazo, A, Fernandez, LM, Fernandez, M: Semi-slant submanifolds of a Sasakian manifold. Geom. Dedic. 78, 183-199 (1999)

27. Chen, BY: Some pinching and classification theorems for minimal submanifolds. Arch. Math. 60, 568-578 (1993)

28. Nolker, S: Isometric immersion of warped products. Differ. Geom. Appl. 6, 1-30 (1996)

29. Wan, PJ, Yi, CW: Coverage by randomly deployed wireless sensor networks. IEEE Trans. Inf. Theory 52, 2658-2669 (2006)

\section{Submit your manuscript to a SpringerOpen ${ }^{\circ}$ journal and benefit from:}

- Convenient online submission

Rigorous peer review

- Immediate publication on acceptance

- Open access: articles freely available online

- High visibility within the field

- Retaining the copyright to your article 